\title{
El mundo en un espejo. Percepciones campesinas de los cambios ambientales en el Occidente de México
}

\author{
Peter R. W. Gerritsen*, María Montero C.** \\ Y PEDRo Figueroa B.***
}

\begin{abstract}
An understanding of the current policies that conjugate the conservative interests with the develompental demands needs an analysis of the sustainable development concept. This study examines how the environmental change is perceived by a farmer comunity located in a Biosphere Reserve. We observe how the policies promoted by the globalising development tear down progressively the universe of meanings of the farmers. Thus, their particular view of the natural, productive and social cycles goes through a crisis, creating a dependence based on the incomprehension and ignorance of external factors. This analysis leads us to address some ideas for the management of protected natural areas, taking into consideration all the actors, interests and notions involved in order to achieve an integrating and communicative sustainable development.
\end{abstract}

Keywords: rural sociology, local knowledge, sustainable development, management of protected natural areas.

\section{Resumen}

Un análisis del concepto de desarrollo sustentable es fundamental para comprender la dinámica de las políticas actuales que deben conciliar los intereses conservacionistas con las demandas desarrollistas. Nuestro estudio analiza cómo los campesinos de una comunidad rural, que está situada en una Reserva de la Biosfera, perciben el cambio ambiental. El trabajo observa cómo las políticas impulsadas por el desarrollo globalizador van desestructurando progresivamente el universo de significados de los campesinos, de manera que entra en crisis su particular concepción del ciclo natural, productivo, y social, a la vez que se genera y ocasiona un rol de dependencia basado en la incomprensión e ignorancia de los agentes externos. El análisis nos conduce a discutir algunas ideas para la gestión de áreas naturales protegidas donde se tomen en cuenta todos los actores, intereses y concepciones implicadas para conseguir un desarrollo que sea sustentable a la vez que integrador y comunicativo.

Palabras clave: sociología rural, conocimiento local, desarrollo sustentable, gestión de áreas naturales protegidas.

\footnotetext{
*Universidad de Guadalajara. Correo-e: petergerritsen@cucsur.udg.mx

** Universidad de Barcelona. Correo-e: mariacastellana@hotmail.com

*** Universidad de Guadalajara. Correo-e: pfigueroa@cucsur.udg.mx
} 


\section{Introducción}

Deterioro ambiental, pérdida de biodiversidad, contaminación de agua y suelo, deforestación: los problemas ambientales han cobrado gran importancia desde de los años setenta debido a la magnitud en que se nos han manifestado, así como al reconocimiento de su impacto negativo sobre el bienestar de la humanidad (Primack et al., 1998). Como consecuencia, con el fin de frenar estos problemas, durante los últimos años ha surgido una tendencia a escala mundial, y concretamente también en México, que considera la participación ciudadana como un aspecto primordial para el diseño de políticas públicas (Chambers, 1983, 1997; Gutiérrez, 2000; INE, 2000). Sin embargo, es preciso tener en cuenta -a la hora de realizar políticas públicas, intervenciones o toma de decisiones- que diferentes actores mantienen percepciones divergentes, dependiendo de un conjunto de factores, como el nivel socioeconómico, las estrategias de subsistencia, la afiliación política o el bagaje cultural. Es en este sentido que se ha vuelto más complejo el proceso para generar una participación social que sea integradora a la vez que efectiva. Además, como consecuencia, resalta la necesidad de conocer las diferentes percepciones para poder impulsar políticas públicas basadas en la participación ciudadana (Lazos y Paré, 2000; Long y Long, 1992; Gerritsen, 2002).

En este trabajo presentamos, de manera general, las percepciones del cambio ambiental de los campesinos de la comunidad de El Saúz, la cual está ubicada dentro de los límites de la Reserva de la Biosfera Sierra de Manantlán (RBSM), en el occidente de México. A partir del concepto campesino del cambio ambiental, analizaremos la influencia de las decisiones políticas y económicas extracomunitarias sobre la configuración de una determinada percepción y valoración del ambiente por parte de los agentes locales.

Como nuestro estudio se realizó en el contexto de una área natural protegida, entran en juego actores y posicionamientos muy diversos ante una determinada realidad ambiental. Por un lado, encontramos la visión, intereses y exigencias del personal de la Dirección de la Reserva de la Biosfera Sierra de Manantlán (DRBSM) de la Comisión Nacional de Áreas Naturales Protegidas de la Secretaría de Medio Ambiente y Recursos Naturales (Conanp/ Semarnat) que trabaja conjuntamente con el Departamento de Ecología y Recursos Naturales (DERN) del Instituto Manantlán de 
Ecología y Conservación de la Biodiversidad (Imecbio) de la Universidad de Guadalajara en la gestión y manejo de la reserva de la biosfera (Jardel, 1992; Imecbio, 2000a). Por otro lado, tenemos la perspectiva, prioridades y demandas de los habitantes del área natural protegida (Gerritsen, 1995, 1998a, 1998b, 2002; Partida, 2001). Son, como señala Anne Whyte, puntos de vista divergentes que apuntan hacia direcciones aparentemente opuestas: “el punto de vista del interior está caracterizado por el hábito y por una larga experiencia, a menudo asociada a una cierta inaptitud para efectuar transformaciones rápidas. Está considerada como personalizada y subjetiva. En contra, el punto de vista del exterior está asociado al desarrollo, a la acción y a la objetividad, enfrentado con la tradición interior y con la resistencia al cambio" (MAB-UNESCO, 1978: 13).

A continuación presentamos algunas reflexiones teóricas para después presentar nuestro estudio de caso y finalmente discutirlo.

\section{Posicionamiento teórico: percepciones del cambio ambiental}

Es necesario contextualizar el análisis de la percepción de los campesinos dentro del marco de las decisiones impuestas por el desarrollo global y, en nuestro caso, por la creación de una reserva de la biosfera en 1987, cuya gestión pretende conciliar las políticas conservacionistas con los intereses desarrollistas (Jardel, 1992; Imecbio, 2000a; Semarnap, 1996). Esta primera idea se enlaza con el concepto de desarrollo sustentable, considerado actualmente de gran relevancia (INE, 2000; Gutiérrez, 2000). El término busca conjugar la mejora de la calidad de vida de una comunidad rural con la conservación de sus recursos naturales a largo plazo. De manera que, en el contexto de un área que está a la vez habitada y protegida, el desarrollo sustentable emerge con el imperativo de conocer y poder conciliar los diferentes puntos de vista de los actores implicados (Gerritsen, 2001, 1995).

La multiplicidad de perspectivas existentes ante una determinada realidad ambiental nos llevó a plantear la investigación a partir de dos fundamentos teóricos: una definición abierta de cambio ambiental y la idea de que la percepción del ambiente es heterogénea y variable gracias a su propia naturaleza intersubjetiva (Lazos y Paré, 2000). De manera muy general, entendemos el cambio ambiental como un término que indica la transición entre dos situaciones, cuyo resultado puede ser considerado de menor o mayor valor que el inicial (Primack et al., 1998). Esta definición 
flexible permite abarcar su carácter de representación ecológica a la vez que social, puesto que responde a construcciones que reflejan un juicio sobre el acceso y el uso de los recursos naturales (Gerritsen, 2002). Por esta razón, siguiendo las líneas propuestas por Anne Whyte, consideramos que: "la expresión 'percepción del ambiente' significa la toma de conciencia y la comprensión del medio por el individuo en un sentido amplio" (MAB-UNESCO, 1978:18).

En la percepción es fundamental el papel recreativo del sujeto y del colectivo que no sólo captan, sino también entienden, deciden y actúan de una determinada forma sobre su entorno inmediato. Además, el acto de percibir no es homogéneo, depende de variables personales, culturales, sociales, e incluso económicas o políticas, que determinan el mundo percibido subjetivamente. Este proceso implica conocimiento y organización, los valores que son puestos en el ambiente, las preferencias y selecciones (Godínez y Lazos, 2001) y, en definitiva, varía y evoluciona en el tiempo según el contexto y las necesidades particulares (Mendras, 1970; Gerritsen, 2002).

\section{Posicionamiento metodológico: diseño del estudio}

Entre este conjunto de fundamentos teóricos es necesario, de manera breve, esbozar el tipo de estudio que realizamos. El análisis que presentamos a continuación está basado en datos recogidos durante el verano de 2001. La investigación se realizó con una metodología cualitativa, trabajando con un número reducido de estudios de caso (18 individuos), elegidos a partir de tres variables que pretendían abarcar una muestra representativa de la población real: género (hombre/mujer), edad (joven: menor de 25 años/adulto: entre 25 y 50 años/anciano: mayor de 50 años), y actividad desempeñada (campesino ejidatario/campesino avecindado/ganadero/artesano/otras). Los datos se recopilaron a través de entrevistas individuales de tipo semi-estructurado y abierto, aunque en algunos casos mantuvimos pláticas con varias personas simultáneamente (Bernard, 1988; Geilfus, 1997). Con la finalidad de obtener la máxima información, también conversamos con algunos agentes externos clave, influyentes en la comunidad: maestros, párroco, promotores del DERN-Imecbio, personal de la DRBSM-Conanp. 


\section{3. Área de estudio: la comunidad de El Saúz}

La comunidad de El Saúz es una de las cinco comunidades que forman parte del ejido Platanarillo, Municipio de Minatitlán, Colima (véase el mapa I). El Saúz presenta una topografía muy accidentada y una altitud que varia entre los 900 y 1,800 metros sobre el nivel del mar. El clima es cálido subhúmedo, con lluvias de junio a octubre y con presencia de fuertes vientos.

\section{Mapa I}

Ubicación del ejido de Platanarillo en la Reserva de la Biosfera Sierra de Manantlán

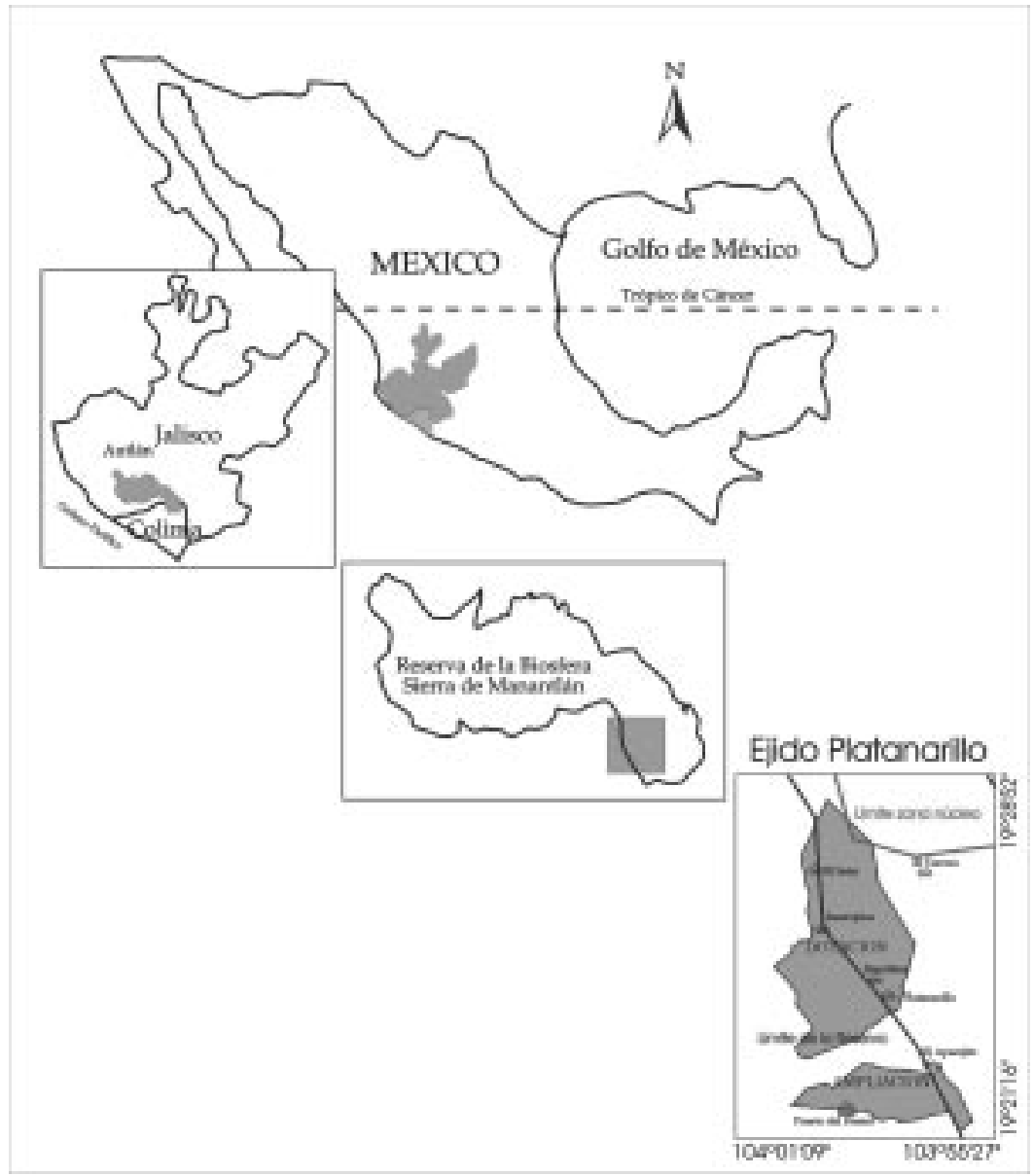


El ejido se fundó en 1945, con una dotación de 1,664 hectáreas, con una ampliación de 1,363 adicionales en 1970, y actualmente consta de 3,027 hectáreas. Por el ejido atraviesa la carretera Colima-Minatitlán, sin embargo, para tener acceso a la comunidad de El Saúz, si no se posee automóvil es necesario caminar $2 \mathrm{~km}$ por un camino de terracería, a partir del crucero de Rastrojitos.

La comunidad alberga unas 36 familias (con un total de 200 habitantes), todas mestizas. La mayoría es ejidataria dedicada principalmente a la producción de maíz para autoconsumo (practicando la roza, tumba y quema), complementada con la ganadería (con un manejo extensivo) con hatos que no sobrepasan las 20 cabezas, la artesanía de otate (Otatea spp.), la recolección de algunos recursos no maderables y la migración temporal a las ciudades del estado de Colima y a los Estados Unidos (Imecbio, 2000b). Una característica importante de El Saúz es que $90 \%$ de los habitantes son artesanos o tienen una vinculación con la cooperativa de artesanos del otate creada en 1996 (Figueroa, 1999).

El Saúz está considerada por dependencias gubernamentales como marginada, presentando un índice de marginación medio, escasez de empleo, dominancia de actividades de subsistencia, topografía accidentada, problemas de alcoholismo, migración de jóvenes, dificultades de comunicación y un alto nivel de analfabetismo; a la fecha cuenta con escuela primaria (dos profesores) y con atención a niños de preescolar a través del Consejo Nacional de Fomento Educativo (Conafe) y el Instituto Nacional para la Educación de los Adultos (INEA) en atención a un grupo de secundaria abierta. También existe una pequeña capilla católica, donde se ofrecen misas una vez al mes. Finalmente, es necesario apuntar que la población cuenta con una promotora de salud local capaz de proporcionar primeros auxilios y con visitas mensuales por el médico de la Secretaria de Salubridad y Asistencia (SSA), con servicio de corriente eléctrica, y con un sistema de agua entubada deficiente que genera conflictos en la comunidad (Imecbio, 2000b).

\section{Contexto institucional: la Reserva de la Biosfera Sierra de Manantlán}

El 74\% del territorio del ejido de Platanarillo se encuentra dentro de la Reserva de la Biosfera Sierra de Manantlán (RBSM), la cual es un área montañosa ubicada al sur del estado de Jalisco y al noreste del estado de Colima, con una extensión de aproximadamente 140,000 hectáreas. Fue establecida en 1987, con el fin de conser- 
var la biodiversidad y promover un uso sostenible de los recursos naturales.

La RBSM presenta una topografía accidentada y gran amplitud altitudinal, entre 400 y $2860 \mathrm{msnm}$, y es representativa de las condiciones ecológicas y del rico patrimonio biológico de las montañas de México. La vegetación es muy diversa, debido a la variabilidad de las condiciones fisiográficas y la amplitud altitudinal (Jardel, 1992).

La RBSM se encuentra en los terrenos de 32 ejidos y comunidades indígenas, y alrededor de 80 propiedades privadas, perteneciendo a cinco municipios de Jalisco y a dos de Colima. La población se dedica principalmente a la agricultura de subsistencia (cultivo de maíz y frijol, como ya se mencionó, a través del sistema de roza, tumba y quema), frecuentemente en terrenos con altas pendientes y pedregosidad. La ganadería extensiva se desarrolló en la mayor parte de la RBSM, mientras que la actividad forestal comercial se encuentra suspendida en toda la Sierra de Manantlán, a excepción del ejido El Terrero. Asimismo, presenta una problemática social y agropecuaria representativa para el país, en cuanto a conflictos en la tenencia de la tierra, bajos rendimientos por hectárea, ganadería extensiva, una marcada marginación social y falta de organización campesina, hechos que provocan una presión mayor sobre los recursos naturales (Imecbio, 2000a; Warman, 2001).

Finalmente, en las reservas de la biosfera, la integración de la conservación y el desarrollo social es el aspecto central. La investigación científica, por su parte, es considerada como el factor que aporta las bases teóricas y los elementos técnicos para fundar el manejo del territorio, sus recursos naturales y la conservación de la biodiversidad. Esto integra las tres funciones básicas de las reservas de la biosfera, siendo la conservación, el desarrollo social y la investigación y educación. En ese sentido, se concibe a las reservas de la biosfera como modelos experimentales o proyectos pilotos de un estilo de desarrollo social y gestión de los recursos y el ambiente basados en la conservación ecológica (Jardel, 1992; Jardel et al., 1996; Imecbio, 2000a, 2000b; cfr. Gerritsen, 2002).

\section{Percepciones campesinas del cambio ambiental}

Son varios los cambios ambientales percibidos por los habitantes de El Saúz, las causas que se les atribuyen y las soluciones que proponen ante su particular situación. De entrada, es interesante 
destacar que la mayoría de los cambios remarcados en el Diagnóstico Integral y Plan Comunitario de Manejo de los Recursos Naturales del Ejido de Platanarillo (Imecbio, 2000b; cfr. Toledo y Bartra, 2000), realizado en 1998 por el personal de DERN-Imecbio y de la DRBSM, no fueron significativos para los habitantes que se entrevistaron durante este estudio. Como muestra la gráfica 1, los principales cambios ambientales percibidos por los campesinos de El Saúz han sido la falta de agua y de otate, el aumento de plagas, la disminución de la fertilidad de los suelos por un lado, y de los árboles y variedad de cultivos por el otro. Estos aspectos fueron mencionados por más de la mitad de los entrevistados, en contraposición al resto que permanecieron casi inadvertidos, como los incendios, la disminución en la diversidad de plantas y animales o el aumento de la erosión, temas que destacan porque están considerados de gran preocupación por parte de muchos conservacionistas contemporáneos (Primack et al., 1998). Como una primera aproximación, la gráfica es representativa del marco genérico que envuelve las concepciones campesinas de la realidad, a la vez que insinúa la distancia respecto de las visiones oficiales sobre la situación ambiental de la zona. En los apartados siguientes analizaremos a fondo los cambios ambientales más importantes percibidos por los campesinos de El Saúz.

\section{"El agua es la vida"}

El principal cambio ambiental apuntado por los habitantes de El Saúz es la falta de agua. La disminución de las lluvias y del caudal de los ríos y arroyos en general, ha sido el tema más insistido. " $E l$ agua es la vida", nos comentaban en las entrevistas, pues es un elemento que abarca todos los ámbitos de su vida: el consumo personal, el riego de la tierra, la bebida para el ganado y el gasto para lavar, cocinar y asearse. De esta manera, los campesinos afirmaban haber notado una progresiva disminución del agua disponible en la comunidad a lo largo del tiempo, apuntando que, desde que su memoria alcanza a recordar, cada año ha escaseado más. Este descenso paulatino del agua se ha percibido principalmente a través de la reducción de lluvias, y ha provocado que el agua sea la principal preocupación y tema de debate en El Saúz (Partida, 2001).

En general, los campesinos argumentaban dos causas fundamentales que explicaban la falta de agua: una primera, de tipo productivo, y una segunda relacionada con el carácter del ser 


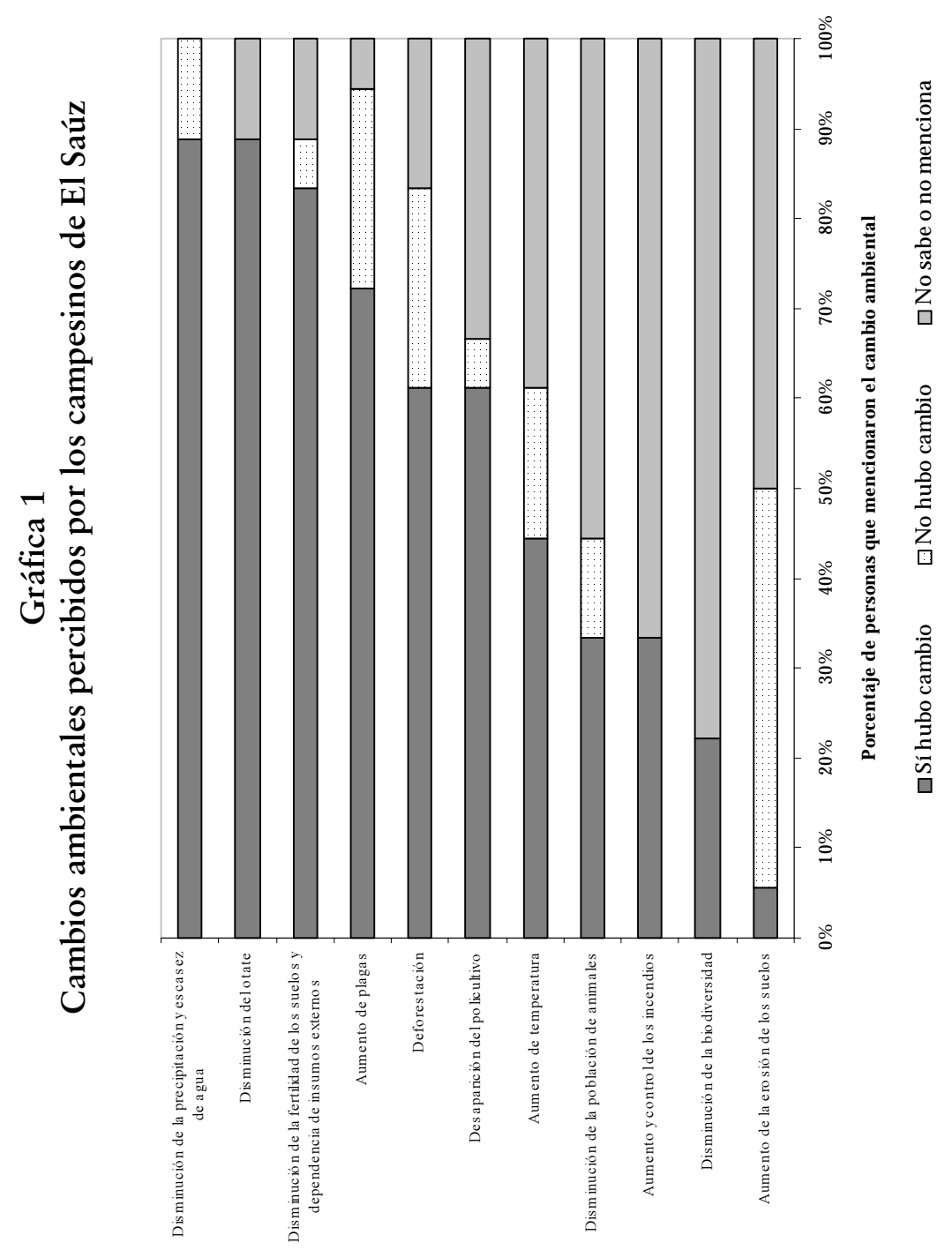


humano. Las causas reflejan la asociación entre la escasez de agua con tala de árboles por un lado, y con el desperdicio y falta de conciencia humana por el otro. Esta segunda explicación está directamente vinculada con un conflicto del abastecimiento y distribución del agua. "Nadie quiere reconocer que tira el agua", apuntaba una joven de El Saúz, "la falta de agua es porque la desperdiciamos, porque en ese tiempo no le hace falta el agua y luego cuando le hace falta, vienen renegando."

\section{La naturaleza en ciclos: "la falta de otate"}

El segundo cambio ambiental más mencionado es la disminución de otate. Éste es un producto básico en la vida de El Saúz, pues constituye la materia prima para realizar artesanías (canastas pizcadoras, chiquihuites y tortilleros) y, aunque actualmente haya disminuido el trabajo del otate, sigue siendo una fuente de ayuda económica importante, sobre todo en el caso de los avecindados, que no pueden acceder a la posesión de tierras. A su vez, es interesante remarcar que todos los habitantes de El Saúz conocen la cooperativa de artesanos "El Saúz", creada en 1996 con la ayuda del DERN-Imecbio y la DRBSM, para apoyar la organización y comercialización de las artesanías.

En general, la mayoría de los campesinos narraba con normalidad la actual escasez de otate, afirmando que la planta se había secado porque su ciclo natural así lo determinaba y volvería a crecer en siete años. Así pues, casi la totalidad de los entrevistados aseguraba que la falta de otate era un hecho temporal y natural que forma parte del ciclo de vida de la planta, por lo que sólo podían esperar que la naturaleza cumpliera su curso. "Dicen que se secó”, comentaba una señora, "pues que tiene un ciclo y cada veinte o veinticinco años se seca, antes también hubo un tiempo que estaba seco y ahorita ya le volvía a tocar. Y pues tarda siete años en volver a crecer." Paralelamente, algunas personas también apuntaron causas productivas a la falta de otate, como la excesiva explotación o la dificultad para que la planta creciera, porque el ganado se había comido los retoños antes de que se cercara la principal zona de extracción de otate en la comunidad en 1998. 


\section{Reajustando la labor}

La tercera transformación ambiental más mencionada durante las entrevistas fueron todos los cambios relacionados con "la labor", es decir, con el trabajo de la tierra en su sentido más amplio. Los cambios en la labor abarcan desde la entrada de los agroquímicos, la disminución de la fertilidad de la tierra, el aumento de plagas, la desaparición de los policultivos, hasta el aumento de la ganadería.

En primer lugar, una diferencia fundamental en el modo de "hacer la labor" es la necesidad de utilizar insumos externos para conseguir productividad. Introducidos en los años setenta por programas oficiales y por el rumor en torno a los primeros campesinos que los probaban, los agroquímicos prometían reducir el trabajo, aumentar la productividad de la tierra y poder sembrar mayor cantidad de terreno. De modo que su paulatina penetración no sólo conllevó un cambio en los hábitos y técnicas utilizados por los campesinos, sino también toda una alteración en la concepción de la labor: cambiaba la manera de hacerla y las expectativas que de ella podían esperar (Hewitt de Alcántara, 1984; $c f r$. van der Ploeg, 1997). En resumen, los agroquímicos obtuvieron resultados positivos y esperanzadores durante las primeras temporadas, alimentando la confianza de los campesinos. No fue hasta el cabo de varios años que empezaron a percibir disminución en la productividad de la tierra; pero el proceso de desmitificación de unos productos que estaban reconocidos oficialmente y que habían dado buenos resultados hasta la fecha fue lento. "Antes decían que con la pura guadaña ya crecía el maíz", nos comentaba una joven de El Saúz; “empezaron a poner líquido por comodidad, por lo fácil y lo rápido. Y luego ya metieron el fertilizante a la vez para que saliera buen maíz y como les salió más, pues lo siguieron usando. Pues como el nixtamal, ¿quién lo molería a mano habiendo el molino? [...] Y pues ahorita ya el maíz no se daría sin el fertilizante porque la tierra ha perdido fuerza."

Íntimamente relacionado con la dependencia de insumos externos, encontramos el aumento de plagas. Cuando los campesinos hablan de la baja fertilidad de los suelos, apuntan hacia dos direcciones: por un lado, el desgaste provocado por el uso de herbicidas y fertilizantes y, por otro lado, la intensificación del trabajo sobre una misma área desde el parcelamiento de los terrenos comunales a principios de los años setenta. Este último factor 
es determinante en la cadena de cambios ambientales percibidos en El Saúz, los campesinos lo mencionan repetidamente como un referente temporal que marca un antes y un después en la vida de la comunidad.

La repartición individual de parcelas fue el inicio de un proceso que concluiría en 1994 con la distribución (a nivel individual) de los títulos de propiedad por el Programa de Certificación de Derechos Ejidales y Titulación de Solares Urbanos (Procede). El parcelamiento que se realizó dentro de este programa se puede considerar el paso más reciente hacia la propiedad individual, terminando con un largo periodo de trabajo comunal y con la posibilidad de desmontar cada temporada nuevos terrenos que contenían suelos ricos en nutrientes y vegetación. Teóricamente, durante la fundación del ejido, a cada ejidatario le correspondían diez hectáreas, aunque en la práctica en aquel tiempo el parcelamiento estuvo en función de la posibilidad económica para cercar el territorio, hecho que provocó acaparamiento de tierras y, en definitiva, una desigual distribución de los terrenos (Imecbio, 2000b; $c f r$. Warman, 2001). Desde ese entonces la cuestión de la tenencia de la tierra es un punto no resuelto entre los habitantes de El Saúz, que, a partir de 2002 se está intentando solucionar a través de la solicitud de la repartición de una parte comunal del territorio del ejido para equilibrar el acceso a la tierra de todos los ejidatarios.

Esta situación evolucionó de la mano de la primera y única ampliación del ejido, también en los años setenta, que otorgó tierra a buena parte del grupo avecindado de la comunidad (Imecbio, 2000b). Además, el proceso de apropiación de tierras interactuó con la creciente aparición de la ganadería durante los primeros años de la década de los ochenta. El aumento de la ganadería fue impulsado desde los organismos oficiales, con créditos y ayudas, que facilitaban la obtención de pastos exóticos y cabezas de ganado bovino principalmente (Louette et al., 1997, Toledo, 1990; Warman, 2001). En este sentido, es importante destacar la relación entre la concesión de los títulos de propiedad y la disminución del ganado caprino y porcino, pues éstos necesitan libertad de movimiento, mientras la delimitación de parcelas implicaba la reducción de la tierra disponible por persona y fomentaba un tipo de ganado más estático o estabulado. Además, esta situación se vio reforzada porque a finales de los noventa las autoridades oficiales empezaron a presionar para que no hubiera animales sueltos en las comunida- 
des, y a la vez los intermediarios exigieron carne que cumpliera requisitos sanitarios. De esta manera, el ganado bovino se fue imponiendo en detrimento del ganado porcino y caprino, casi inexistente actualmente en la comunidad.

Así pues, el parcelamiento definitivo del territorio en 1994, con la acreditación oficial a través del título de propiedad, resultó ser el momento clave más reciente en la historia de El Saúz que, como apuntábamos anteriormente, conllevó cambios en la manera de concebir "la labor". La nueva coyuntura no permitía respetar el periodo de barbecho necesario para la recuperación de la tierra, de manera que el tradicional sistema de roza, tumba y quema y la concepción del ciclo agrícola que alberga, perdieron su significado. "Antes era mejor", exclamaba un anciano ejidatario, "porque uno podía sembrar donde quisiera y ya tumbaba un pedazo de monte allá arriba y sí se daba bien el maíz. Ya luego no le dejaron a uno hacerlo [...] Es que la tierra necesita recuperarse, ya ahorita uno nomás puede sembrar en su parcela, y pues icuándo descansa la tierra!” A esta situación, cabe añadir la aparición de las ayudas de Procampo en 1994 que, al estar vinculadas al trabajo en una parcela específica, intensifican un desgaste de los terrenos (cfr. Ortiz, 2001). ${ }^{1}$ Además, esta situación se complica con las leyes forestales, que prohíben cualquier cambio de cobertura vegetal sin los permisos correspondientes dentro de los límites de la RBSM (Imecbio, 2000a).

Finalmente, los campesinos relacionan la disminución de la fertilidad de los suelos con la desaparición del poli-cultivo y la presencia de los agroquímicos. Hoy en día encontramos básicamente cultivos de maíz en El Saúz, mientras que en los años setenta existían cultivos paralelos que ayudaban al autoabastecimiento: frijol, jitomate, chile, caña, plátanos, café, mangos, mameyes, ejotes, pepinos, calabazas, rábanos, papayos, guayabos, etc. Este impulso del monocultivo en detrimento del poli-cultivo está también relacionado con las ayudas del programa gubernamental Procampo, que no sólo están vinculadas al trabajo en una parcela específica, sino también al cultivo de productos básicos específicos (maíz, soya, sorgo, trigo, cebada, arroz, principalmente) (Ortiz, 2001). Es interesante destacar que también se asocia la poca variedad de cultivos a la manera del ser humano: la falta de perse-

${ }^{1}$ El Procampo es un programa gubernamental que surgió a principios de los años noventa, y está destinado a ofrecer apoyos a los productores de cultivos básicos (maíz, soya, sorgo, trigo, cebada, arroz, frijol, etcétera) de manera que éstos se vayan insertando paulatinamente en la dinámica del libre mercado. 
verancia por un lado, los robos por otro, y finalmente el temor de fracasar: "tenemos el miedo de arriesgar lo poquito que uno tiene para que luego no pegue".

En resumen, entre los principales argumentos que explican los cambios en la labor están el uso de agroquímicos y la intensificación del cultivo en la misma parcela; esto último resultó ser un hecho que vino a reforzar la creación de la RBSM a través de las reglas administrativas referente al uso del territorio (Imecbio, 2000a). Estos hechos, junto con la disminución de la precipitación y la sequía, complican la calidad de los suelos, dibujando un círculo dantesco en la vida de los campesinos: de la dependencia de insumos externos al aumento de plagas, de la baja fertilidad de los suelos a la desaparición del poli-cultivo y de nuevo al inicio, que está consolidando cada vez más una creciente dependencia que incluye una pérdida de conocimientos empíricos.

\section{"La Forestal": fin a la tala de árboles}

La deforestación es un último aspecto que resulta interesante analizar porque, en contraposición a la visión oficial (Imecbio, 2000a), los campesinos aseguran que la tala de árboles dentro del ejido es en la actualidad insignificante. La disminución de la deforestación está asociada a la creación de "la forestal" aproximadamente en la década de los ochenta, mientras que en 1987 fue creada la RBSM. Se utiliza el término "la forestal" en un sentido algo ambiguo, englobando bajo un mismo concepto organismos muy diversos (las autoridades municipales, el gobierno estatal, la vigilancia desde la DRBSM, hasta el control de miembros procedentes del DERNImecbio o de la Universidad de Guadalajara en general) que, bajo la perspectiva campesina, tienen en común el control de la deforestación.

De esta manera, los campesinos nuevamente apuntan hacia dos direcciones para explicar el elevado índice de deforestación antes de los ochenta. Por un lado, factores productivos, como la existencia de la propiedad comunal y del sistema tradicional de roza, tumba y quema, que llevaban a desmontar terrenos nuevos cada dos o tres ciclos productivos, además de la necesidad de abastecerse de leña para poder cocinar. Por otro lado, el escaso control forestal del gobierno durante esa época. Así pues, a partir del momento que surge "la forestal" y se empieza a controlar la tala de árboles en la comunidad, los campesinos perciben una progresiva reducción de la deforestación que va dejando de ser significa- 
tiva a los ojos de la comunidad. "Aquí casi no tumban árboles", aseguraban, "antes sí, donde había parejos, para sembrar.” Finalmente, un último factor que influyó en el descenso de la deforestación es la Ley Forestal Federal de 1996 que, en términos generales, prohíbe talar vegetación con árboles cuyo diámetro sea superior a diez centímetros, o en su caso, tengan más de cinco años de descanso (Imecbio, 2000a, 2000b). Esta ley ha provocado que los campesinos no dejen crecer los árboles en sus parcelas, con lo que se reduce el tiempo de descanso de la tierra y disminuye su fertilidad.

\section{Las propuestas: pensando el futuro}

Este último subapartado engloba las soluciones aludidas por los campesinos para hacer frente a la situación ambiental en la que se encuentran. En primer lugar, encontramos las propuestas relacionadas con el ahorro del agua, como la concientización de la gente en el cuidado y uso del agua, la ampliación del pozo de la comunidad y la estricta aplicación de las normas del uso del agua. En segundo lugar, la insistencia en la necesidad de aumentar los apoyos del gobierno a la comunidad, pagando a mejor precio el maíz o el salario del jornalero, generando puestos de trabajo, rebajando los costos de los agroquímicos, etc. El tercer grupo incluye soluciones tan diversas como el uso de fertilizantes orgánicos, la capacitación de la población en el uso de los recursos naturales, reforestar y poder aprovechar los árboles que beneficien a la comunidad, y el nuevo intento de diversificar los cultivos. Finalmente, encontramos las propuestas menos mencionadas, como aumentar las medidas de control de las autoridades oficiales, mayor comunicación entre la comunidad y el gobierno y, por último, hacer terrazas o introducir plantas para evitar el deslave. "Necesita uno saber para cuidar la tierra, necesita uno ver, ver y saber cómo hacerlo [...] porque si no cuida uno su tierra, ¿quién la va a cuidar?"

\section{Más allá del cambio ambiental}

Podemos diferenciar un número de aspectos que no están explícitamente relacionados con la percepción del cambio ambiental, pero que influyen en la representación del entorno y de la concepción de la vida en El Saúz. Son cambios endógenos y exógenos que afectan directamente la vida comunitaria y alteran los funda- 
mentos del universo de significación. Por un lado, las transformaciones de El Saúz desde la fundación del ejido en los años cuarenta, y por otro lado, la penetración e influencia de los agentes externos.

\section{Transformaciones de la vida cotidiana}

Son varios los cambios que ha sufrido El Saúz a lo largo del tiempo y que fueron apuntados por los campesinos durante las entrevistas colateralmente a las transformaciones ambientales: desde cambios en la salud personal y alimentación, hasta el aumento de la emigración, la pérdida de conocimiento entre generaciones y, en general, la alteración de costumbres y hábitos tradicionales. Así pues, un primer factor que emergía durante las entrevistas es la mejora de las condiciones de vida de la comunidad. Desde que se fundó el ejido en 1945 hasta la época actual, El Saúz ha padecido varios cambios significativos que han influido en la transformación de su concepción del mundo, trabajo y vida en la comunidad: creación de caminos de terracería y mejora en las comunicaciones, introducción de la electricidad desde mediados de los setenta (lo que va acompañado de la entrada del molino eléctrico, radios, televisores, etc.), la mejora de viviendas a través de un programa gubernamental puesto en marcha también en los setenta, la construcción parcial del entubado del agua a finales de los noventa, etc. También apuntaban como un hecho significativo la llegada de la gente de la RBSM desde mediados de los años noventa (incluyendo bajo el término "gente de la Reserva" a promotores del DERN-Imecbio, personal de la Universidad de Guadalajara y de la DRBSM indistintamente). Finalmente, insistían en que aumenta la emigración de los jóvenes a Colima y a grandes ciudades de Estados Unidos (cfr. Arroyo, 1989).

\section{La llegada de los agentes externos}

A grandes rasgos, entendemos por agentes externos el conjunto de instituciones, organismos y personas ajenas a El Saúz que intervienen o influyen en la vida comunitaria (Chambers, 1997). La definición incluye los medios de comunicación en general, el gobierno estatal y nacional, la DRBSM, el DERN-Imecbio y, en última instancia, las organizaciones religiosas y la escuela. El factor externo más significativo es la presencia del discurso de los medios de comunicación en los comentarios de la mayoría de campesi- 
nos, lo que representa la consecuencia más palpable del proceso de globalización. La información proveniente del exterior transcurre básicamente a través de la radio y, sobre todo, para el género masculino, que tiene mayor oportunidad de escucharla a lo largo del día.

Paralelamente, aunque podemos afirmar una cierta penetración del discurso conservacionista relacionado a la RBSM, la mayoría de los campesinos conocen su funcionamiento y trabajo de manera bastante general y ambigua, o incluso no saben que El Saúz forma parte de ella. Este aspecto es significativo principalmente entre el género femenino (Gerritsen 1998a; Imecbio, 2000a). En este sentido, es necesario apuntar que la gran mayoría de programas impulsados desde la DRBSM y el IMECBIO actúan sobre la población masculina. En general, las mujeres están al margen de los contactos con el personal de la RBSM y las autoridades oficiales, mientras son quienes mantienen la relación con la escuela y el párroco (Imecbio, 2000a; Gerritsen, 1998b). A su vez, el discurso ambientalista también se introduce en la comunidad a través de hombres que han ocupado cargos políticos o han tenido contacto frecuente con las autoridades oficiales, o a través de jóvenes que han podido continuar sus estudios fuera de El Saúz y conocen el discurso ecologista actual. También es necesario tener en cuenta dos últimos aspectos: por un lado, la presencia de la escuela, que trabaja siguiendo los lineamientos oficiales y, por otro, el hecho de que al menos la mayoría de la población es católica practicante y acude a las misas oficiadas por el párroco una vez al mes.

\section{Discusión: el universo de la ruralidad}

A lo largo del estudio, las percepciones de los campesinos de El Saúz han aparecido enmarcadas dentro de un contexto mucho más amplio que trasciende incluso las fronteras de México. El análisis ha ido configurando una concepción del deterioro ambiental que a la vez diverge y converge con la idea de los agentes externos. Mientras el discurso oficial habla del problema ambiental y sus repercusiones ecológicas, los campesinos hablan de los hechos y de la realidad natural ante la cual deben encontrar la manera de adaptarse para sobrevivir. Es una diferencia de matices que alberga en su seno distintos universos de significación. En este último apartado queremos presentar la concepción general 
de los habitantes de El Saúz en relación con esta coyuntura nacional e internacional que se ha ido insinuando durante el trabajo.

En primer lugar, para los campesinos la naturaleza está en continuo movimiento, hecho que genera una mirada ambivalente hacia el entorno. Por un lado, son conscientes de que la naturaleza no es una fuente inagotable de recursos y defienden la necesidad de respetar los ciclos naturales para permitir su recuperación. Pero por otro lado, esta visión de un entorno cambiante provoca que algunos aspectos del deterioro se tornen invisibles, pues se convierten en imperativos del camino del tiempo, al igual que las mejoras en las comunicaciones, la electricidad, etc.

A esta ambivalencia latente en la percepción ambiental de la gente de El Saúz es necesario sumarle la estrecha relación que mantienen con la naturaleza. La vida de un campesino está construida a partir de las adaptaciones a la naturaleza y a sus alteraciones, de manera que todas sus actividades giran entorno a ella, ya sea directa o indirectamente (Van der Ploeg, 1997). Ante esta situación, han desarrollado una visión esencialmente práctica de la vida, de manera que tanto el conocimiento como la actitud ante la degradación están impregnados de esta concepción práctica, que se pregunta cómo adaptarse, y no por qué se ha dado el cambio. Este conocimiento práctico conlleva también una actitud reacia ante innovaciones y cambios impulsados desde el exterior cuyos resultados no conocen personalmente ni a través de otros campesinos. A estos aspectos debemos sumarle el hecho de que la magnitud del riesgo y el valor de una pérdida son muy grandes en una situación de pobreza como la que encontramos en El Saúz. En este sentido, es importante apuntar que, a lo largo del estudio, El Saúz se ha ido perfilando como un microcosmos donde se manifiestan muchos aspectos característicos de la existencia humana. De esta manera, si bien no podemos extrapolar esta investigación a campos más amplios, puede servir de guía para comprender ciertas coyunturas actuales, como si estuviéramos mirando el reflejo del mundo en un pequeño pedazo de espejo. Hechos como la dificultad de cambiar hábitos y costumbres, el imperativo de obtener resultados inmediatos, el miedo al fracaso, la idealización del pasado, no valorar aquello que no representa un esfuerzo personal, la dificultad de percibir cambios a largo plazo, o los problemas para confiar y trabajar en grupo, son elementos vigentes en muchas sociedades contemporáneas (Giddens, 1984).

Paralelamente, otro factor decisivo en las percepciones ambientales es la presencia de un contexto nacional que no ha fo- 
mentado el conocimiento en el mundo rural, relegándolo a la categoría de ignorante y, por consecuente, dependiente. Este último sentimiento está generalizado en la comunidad, y aparecía en las pláticas acompañado de la necesidad de aprender en el sentido más amplio, de ahí que una de las demandas más insistidas haya sido la capacitación y orientación. A su vez, esta sensación de dependencia provoca que los habitantes de El Saúz no vislumbren verdaderas soluciones sin una intervención externa, y refuerza la poca confianza que tienen en sí mismos como grupo social con poder de cambio.

Es necesario, también, estructurar el vínculo con la historia de México. La corrupción, por un lado, y el fracaso (relativo) de la Revolución Mexicana para los campesinos, por el otro, han conjugado los elementos clave de la profunda desconfianza ante el gobierno y las autoridades en general (Warman, 2001). Además, este contexto histórico ha reforzado el imperativo de obtener resultados inmediatos, ha desacreditado las autoridades locales (ejidales y municipales) y ha motivado el incumplimiento de leyes y normativas (hecho que explicaría, en parte, el problema interno de no poder regular el desperdicio de agua). A la vez, existe una sensación generalizada entre los campesinos entrevistados de haber sido engañados por las instituciones oficiales como, por ejemplo, en el caso de los agroquímicos.

Ante esta coyuntura, es importante diferenciar que si bien los campesinos entrevistados citan discursos oficiales y se piensan como dependientes de agentes externos para poder salir adelante, existe una profunda desconfianza ante todo aquello proveniente del exterior. Es imprescindible tener claro este doble papel de los agentes externos: por un lado, poseen los recursos y conocimientos necesarios para mejorar la situación, por otro lado, han quedado desacreditados por precedentes históricos. En este contexto se comprende la exigencia de obtener resultados palpables y a corto plazo de los programas de apoyo realizados desde el exterior, para reacreditar las intenciones externas a los ojos de la comunidad.

A este entramado de relaciones y expectativas, debemos sumarle la diferente percepción e importancia de los cambios ambientales entre los campesinos y los agentes externos. Como comentamos en los resultados, es significativo que los habitantes de El Saúz no hayan percibido la ganadería o la erosión como uno de los principales cambios naturales, como se insiste, por ejemplo, en el Diagnóstico Integral y Plan Comunitario de Manejo de Re- 
cursos Naturales. Esta distinta percepción esconde concepciones que van más allá de la naturaleza, su uso y aprovechamiento, a la vez que remite a prioridades y necesidades diferentes.

La lejanía entre la visión de la comunidad en general y los diferentes agentes externos está reflejada también en la invisibilidad de la fecha de creación de la RBSM (1987), de la cual forma parte El Saúz. Al inicio de la investigación, la propusimos como posible fecha significativa de manera que la preguntábamos indirectamente durante las entrevistas. Pero 1987 resultó ser una fecha intrascendente para el conjunto del campesinado entrevistado, y la única mención de la existencia de la RBSM se remonta tan sólo cinco o seis años atrás (1996/1995), momento más cercano a la creación de la Secretaría de Medio Ambiente y Recursos Naturales (Semarnat) en 1994 y a la época en que el DERN-Imecbio destinó un promotor en esa zona. En contraste con la invisibilidad de esta fecha, un momento muy mencionado fue el parcelamiento de tierras durante los años setenta. Como exponíamos en los resultados, es un punto de inflexión en la historia de la comunidad que culmina en 1994 con la entrega de los títulos de propiedad por parte de Procede. Así pues, a partir de los setenta, los campesinos empiezan a sentirse propietarios de sus terrenos, con lo que adquieren total libertad de actuación. Este hecho conduce a una progresiva primacía del interés individual por encima el colectivo, y a un aumento del individualismo en el seno de la comunidad. A este contexto cabe sumar los programas gubernamentales, principalmente a partir de los años setenta, dirigidos a impulsar un aumento de producción per se, a través de la vinculación de las ayudas al hecho de demostrar que se estaba trabajando la parcela, lo que conducía al campesino a desmontar su tierra para mostrarlo de manera inequívoca ( $c f r$. Lazos y Paré, 2000).

\section{Conclusiones}

Quisiéremos remarcar que nos encontramos ante una retórica internacional que asocia el actual discurso de desarrollo sustentable con unas políticas globalizadoras y conservacionistas que han ido desestructurando progresivamente la concepción del ciclo natural y productivo de muchas comunidades rurales. Pero esta coyuntura no sólo está haciendo tambalear todo un universo de significación, también está generando un rol de dependencia basado en argumentos que alegan la ignorancia e incapacidad de los campesinos para adaptarse a nuevos tiempos, entiéndase tiempos 
forzosos de un determinado tipo de globalización. En este contexto, surge la gran cuestión de cómo se puede lograr un desarrollo sustentable, a lo cual queremos contribuir con algunas reflexiones.

Para empezar, nos gustaría destacar la necesidad de entender el desarrollo sustentable no solamente en términos ecológicos, sino pensamos que habría que tomar en cuenta otros aspectos como son la equidad, igualdad, inclusión social, raíces culturales, democracia y participación ciudadana, entre otros (Pretty, 1995; Chambers, 1997). Además, opinamos que la sustentabilidad necesariamente debe surgir de las capacidades y conocimientos de los actores locales (Toledo, 2000), como son los campesinos de El Saúz, en nuestro caso, y a través dinámicas en las que el papel de los agentes externos debería consistir más que nada en reforzar los procesos de desarrollo sustentable endógeno (Pretty, 1995).

Obviamente, no existen recetas o prescripciones para impulsar la sustentabilidad. Al contrario, es sumamente necesario analizar para cada contexto local la configuración ecológica, política y socioeconómica específica que determina el tipo de sustentabilidad que se puede lograr. Reconociendo esta heterogeneidad en los ámbitos local y regional, queremos contribuir al debate planteando algunas ideas generales para la gestión y manejo de las áreas naturales protegidas, y donde los mecanismos de participación rebasan su funcionalidad para la conservación (Gerritsen, et al., 1997).

Para empezar, el estudio muestra que existen diferentes perspectivas acerca los grandes temas que les preocupan a los conservacionistas, como son el deterioro ambiental y la conservación de la biodiversidad. Por lo tanto, un primer paso para lograr un desarrollo sustentable es aquel donde todos los puntos de vista sean tomados en cuenta (Gerritsen y Morales, 2001). Esto significa desarrollar espacios sociales de diálogo y de comunicación que impliquen, justamente, encontrarse, conocerse y poder llegar a un consenso en el que todas las partes implicadas se sientan representadas, y donde se concilien, discutan y acuerden las diferentes perspectivas. En otras palabras, habría que desarrollar las “plataformas de discusión” (Röling, 1994). Más importante aún, la puesta en marcha de las plataformas de discusión implica que los grandes asuntos como la sustentabilidad y la conservación de la biodiversidad se conviertan en temas de discusión de orden político y, consecuentemente, en temas de debate y discusión públicos que necesitan de la participación de la ciudadanía para to- 
mar sentido, consistencia y legitimidad, transgrediendo los límites del espacio privilegiado de los científicos y de los políticos. Debido a la existencia de conflictos latentes y activos en muchas regiones y entre muchos actores, proponemos la inclusión de principios de manejo de conflictos en las plataformas de discusión. Significa también que entendemos el proceso participativo, más que como un proceso de aprendizaje mutuo, como un proceso de negociación. Finalmente, cabe señalar que todo lo mencionado hasta el momento implica una revisión a fondo tanto de las ciencias naturales como de las sociales (Gerritsen, 2002).

El desarrollo y la puesta en marcha de un enfoque que cumpla de raíz lo anteriormente discutido para construir un desarrollo que no sólo sea sustentable, sino también contemporáneo, analítico e integrador, de manera que se concilien realmente el desarrollo de una comunidad con la conservación de sus recursos a largo plazo es el mayor desafío con que nos enfrentamos como profesionales en la madrugada de este siglo que apenas comenzó.

\section{Agradecimientos}

Este trabajo fue financiado por el Global Livestock Collaborative Research Support Program (GL-CRSP), Office of Agriculture and Food Security, United States Agency for International Development (USAID).

\section{Bibliografía}

Arroyo A., J. (1989), El abandono rural. Un modelo explicativo de la emigración de trabajadores rurales en el occidente de México, Universidad de Guadalajara, Guadalajara.

Bernard, H. R. (1988), Research methods in cultural anthropology, sage Publications, Newbury Park/London/New Delhi.

Chambers, R. (1983), Rural development: putting the last first, Longman Scientific and Technical, London.

Chambers, R. (1997), Whose reality counts? Putting the first last, Intermediate Technology Publications, London. 
Figueroa B., P. (1999), Organización y comercialización en la comunidad de El Saúz, Ejido Platanarillo, Municipio de Minatitlán, Colima, DERN-Imecbio, Informe Técnico, Autlán.

Geilfus, F. (1997), 80 herramientas para el desarrollo participativo: diagnóstico, planificación, monitoreo, evaluación, Prochalate-IICA, San Salvador.

Gerritsen, P.R.W. (1995), Styles of Farming and forestry. The case of the Mexican community of Cuzalapa, Wageningen Agricultural University, Circle for Rural European Studies, Wageningen Studies on Heterogeneity and Relocalization 1 , Wageningen.

(1998a), Percepciones, conocimientos y actitudes acerca de la Reserva: una aproximación. Estudio de caso de Cuzalapa, Universidad de Guadalajara, CUCSUR/DERN/Imecbio/ PDASM, Informe Técnico, Autlán.

(1998b), “Community development, natural resource management and biodiversity conservation in the Sierra de Manantlán biosphere, Mexico", Community Development Journal, Vol. 33, núm. 4, pp. 314-324.

(2002), Diversity at stake. A farmers' perspective on biodiversity and conservation in western Mexico, Wageningen Studies on Heterogeneity and Relocalization 4, Wageningen.

Gerritsen, P.R.W., S. Graf y S.M. Kreutzer (1997), “Todos juntos, pero no revueltos. Participación, desarrollo comunitario y manejo de recursos naturales en la reserva de la biosfera Sierra de Manantlán en el Occidente de México”, Samaipata, Memorias del taller Enfoques Participativos en Manejo Forestal y de Recursos Naturales, CIAT/DFID, Santa Cruz, Bolivia.

Gerritsen, P.R.W. y J. Morales H. (2001), “Conservación de la biodiversidad. El papel del desarrollo y de la participación local", Sociedades Rurales. Producción y Medio Ambiente, Vol. 2, Núm. 2, diciembre, pp. 87-94. 
Giddens, A. (1984), The constitutions of society. Outline of the theory of structuration, University of California Press, Berkeley and New York.

Godínez, L. y E. Lazos (2001), "Percepciones y sentires de las mujeres sobre el deterioro ambiental: retos para su empoderamiento", en E. Tuñon, Experiencias sobre desarrollo sostenible en América, México.

Gutiérrez N., R. (2000), Introducción al estudio del derecho ambiental, Editorial Porrúa, $3^{\text {a }}$ edn., México.

Hewitt de Alcántara, C. (1984), Anthropological perspectivas on rural Mexico, Routledge and Kegan Paul, London.

Imecbio (Instituto Manantlán de Ecología y Conservación de la Biodiversidad) (2000a), Programa de manejo de la reserva de la biosfera Sierra de Manantlán, México, Semarnap, INE, México.

- (2000b), Diagnóstico integral y plan comunitario de manejo de recursos naturales del ejido de Platanarillo, Municipio de Minatitlán, Colima, Universidad de Guadalajara, Autlán.

INE (Instituto Nacional de Ecología) (2000), Protegiendo al ambiente. Politicas y gestión institucional. Logros y retos para el desarrollo sustentable 1995-2000, Semarnap, INE, México.

Jardel P., E.J. (Coord.) (1992), Estrategia para la conservación de la reserva de la biosfera Sierra de Manantlán, Universidad de Guadalajara, LNLJ, El Grullo.

Jardel P., E.J., E. Santana C. and S.H. Graf M. (1996), “The Sierra de Manantlán Biosphere Reserve: conservation and regional sustainable development", Parks (6) 1, pp. 14-22.

Lazos, E., y L. Paré (2000), Miradas indígenas sobre una naturaleza entristecida. Percepciones del deterioro ambiental entre nahuas del sur de Veracruz, México, IIS, unam/Plaza y Valdés Editores, México. 
Long, N. and A. Long (Eds.) (1992), Battlefields of knowledge. The interlocking of theory and practice in social research and development, Routledge Publishers, London y New York.

Louette, D., P.R.W. Gerritsen y J.J. Rosales A. (1997), La actividad ganadera en la reserva de la biosfera Sierra de Manantlán: un primer diagnóstico, Universidad de Guadalajara, CUCSUR/DERN/Imecbio/PDASM, Autlán.

MAB-UNESCO (1978), La perception de l'environnement: lignes directrices méthodologiques pour les études sur le terrain, Notes techniques du мав 5, Preparé en coopération avec le Scope, unESCO.

Mendras, H. (1970), The vanishing peasant: innovation and change in French agriculture, Cambridge University Press, Cambridge.

Ortiz G., M. G. (2001), El impacto de Procampo en las estrategias campesinas en la comunidad indígena de Cuzalapa, municipio de Cuautitlán, Jalisco, Universidad de Guadalajara, CUCSH, DEPS, DS, Guadalajara.

Partida S., F. (2001), Género, agua, otate y desarrollo comunitario: una investigación en la comunidad El Saúz en la reserva de la biosfera Sierra de Manantlán, México, Universidad de Guadalajara, CUCSH/DEPS/Ds, Guadalajara.

Ploeg, J.D. van der (1997), “On rurality, rural development and rural sociology", in H. de Haan y N. Long (Eds.), Images and realities of rural life. Wageningen perspectives on rural transformations, Van Gorcum Publishers, Assen, pp. 39-73.

Pretty, J.N. (1995), Regenerating agriculture: policies and practice for sustainability and self-reliance, Earthscan Publications Ltd., London.

Primack, R., R. Rozzi, P. Feinsinger, R. Dirzo y F. Massardo (1998), Fundamentos de conservación biológica. Perspectivas latinoamericanas, Fondo de Cultura Económica, México. 
Röling, N.G. (1994), "Platforms for decision-making about ecosystems", in L.O. Fresco, L. Stroosnijder and J. Bouma (Eds.), Future of the land: mobilising and integrating knowledge for land-use options, John Wiley and Sons, Chichester, pp. 386-393.

Semarnap (Secretaría de Medio Ambiente, Recursos Naturales y Pesca) (1996), Programa de áreas naturales protegidas de México 1995-2000, Semarnap/INE, México.

Toledo, V.M. (1990), "El proceso de ganaderización y la destrucción biológica y ecológica de México”, en E. Leff, (Ed.), Ambiente y desarrollo en México, Vol. I, unam, México, pp. 191-227.

- (2000), La Paz en Chiapas. Ecología. Luchas indígenas y modernidad alternativa, Ediciones Quinto Sol/unam, México.

Toledo, C. y A. Bartra (Coords.) (2000), Del círculo vicioso al círculo virtuoso. Cinco miradas al desarrollo sustentable de las regiones marginadas, Semarnap, México.

Warman, A. (2001), El campo mexicano en el siglo XX, Fondo de Cultura Económica, México.

Enviado: 21 de marzo de 2003. Reenviado: 26 de agosto de 2003. Aceptado: 1 de septiembre de 2003. 